\title{
Tendencias mundiales de la implementación de las Normas Internacionales de Información Financiera. Un estudio bibliométrico del período 1989 a 2018
}

\author{
DEIVI DAVID FUENTES DORIA* \\ ANÍBAL TOSCANO HERNÁNDEZ** \\ HÉCTOR GARCÍA ALARCÓN***
}

* Doctor en Ciencias Gerenciales. Universidad Pontificia Bolivariana, Montería, Colombia. E-mail: deivi.fuentesd@upb.edu.co. ORCID: 0000-0002-8798-7156. Google Scholar: https://scholar.google.com/ citations? user $=\mathrm{NfH} 3$ bgEAAAAJ\&hl=es.

** Doctorando en Economía y Empresa. Universidad del Sinú, Montería, Colombia. E-mail: anibaltoscano@unisinu.edu.co. ORCID: 0000-0002-5064-4280. Google Scholar: https://scholar.google.es/citations?user=pwWh2olAAAAJ\&hl=es.

$\star \star \star$ Doctorando en Administración Gerencial. Universidad Cooperativa de Colombia, Montería, Colombia. E-mail: hector.garciaa@campusucc.edu.co. ORCID: 000o-0002-1648-2388. Google Scholar: https://scholar.google.com/ citations?view_op=list_works\&hl=es\&user=YBJRHHQAAAAJ. 


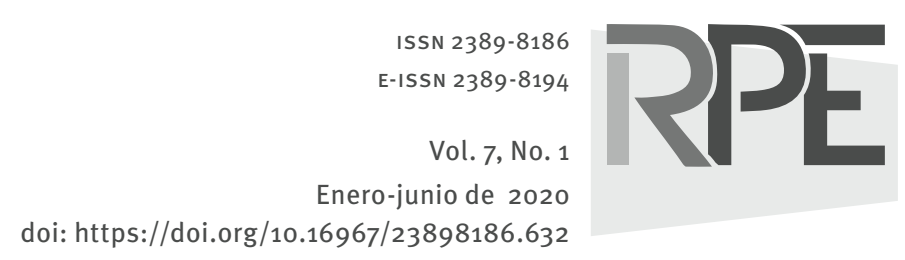

COMO CITAR ESTE ARTÍCULO How to cite this article:

Fuentes, D., Toscano, A. y García, H. (2020). Tendencias mundiales de la implementación de las Normas Internacionales de Información Financiera. Un estudio bibliométrico del período 1989 a 2018. Revista Perspectiva Empresarial, 7(1), 105-121.

Recibido: 08 de julio de 2019

Aprobado: 02 de octubre de 2019
RESUMEN El presente artículo tiene como objetivo describir las principales características bibliográficas de la producción científica mundial relacionada con la implementación de las Normas Internacionales de Información Financiera -NIIF-. A partir de un análisis descriptivo de la evolución cuantitativa de las citaciones, redes de colaboración internacional, productividad de los principales autores y términos frecuentes en las publicaciones científicas.

Se realizó un estudio bibliométrico de revistas científicas indexadas en Scopus a través del software VOSviewer que facilita la visualización de mapas bibliométricos. Lo anterior, permite obtener una visión general de las tendencias de la investigación científica relacionada con la implementación de las NIIF. Este es el primer estudio bibliométrico que informasobrelasprincipales redes decolaboraciónylosclústerestemáticos dela producción científica relacionada con la implementación de estas normas en las organizaciones a nivel mundial. La estrategia de búsqueda empleada identificó 1224 documentos de producción científica que permiten visualizar la importancia de este campo de estudio en la literatura académica, lo que se refleja en un incremento del $99 \%$ en el número de publicaciones anuales entre 1989 y 2018. Dada la rigurosidad de la metodología, y el enfoque global del ámbito de estudio, los resultados del documento facilitan la identificación efectiva de las direcciones de la investigación sobre la implementación de las NIIF en las organizaciones.

PALABRAS CLAVE implementación, análisis bibliométrico, análisis de citaciones, análisis de redes.

\section{World trends in the implementation of International Financial Reporting Standards. A bibliometric study of the period 1989-2018}

ABSTRACT The present paper aims at describing the main bibliographic characteristics of the world's scientific production related to the implementation of International Financial Reporting Standards -IFRS-. This is done from a descriptive analysis of the quantitative evolution of citations, international collaboration networks, productivity of the main authors and frequent terms in scientific publications.

A bibliometric study of scientific journals indexed in Scopus was conducted through the VOSviewer software which facilitates visualization of bibliometric maps. This allows to obtain a general vision of scientific research trends related to the implementation of IFRS. This is the first bibliometric study reporting on the main collaboration networks and thematic clusters of scientific production related to the implementation of these standards in organizations worldwide. The search strategy used identified 1224 scientific production documents allowing to visualize the importance of this study field in academic literature, reflected in an increase of $99 \%$ in the yearly number of publications between 1989 and 
2018. Given the rigor of the methodology and the global approach of the study area, the results make it easier to effectively identify the direction of research on the implementation on IFRS in organizations.

KEY WORDS implementation, bibliometric analysis, citations analysis, network analysis.

\section{Tendências globais na implementação das Normas Internacionais de Informação Financeira. Um estudo bibliométrico do período de 1989 a 2018}

RESUMO Este artigo tem como objetivo descrever as principais características bibliográficas da produção científica mundial relacionadas à implementação das Normas Internacionais de Relato Financeiro -IFRS-. Com base em uma análise descritiva da evolução quantitativa das citações, redes internacionais de colaboração, produtividade dos principais autores e termos frequentes em publicações científicas.

Um estudo bibliométrico de revistas científicas indexadas no Scopus foi realizado por meio do software VOSviewer, que facilita a visualização de mapas bibliométricos. Isso permite uma visão geral das tendências de pesquisas científicas relacionadas à implementação do IFRS. Este é o primeiro estudo bibliométrico que relata as principais redes de colaboração e grupos temáticos de produção científica relacionados à implementação desses padrões em organizações em todo o mundo. A estratégia de busca utilizada identificou 1224 documentos de produção científica que permitem visualizar a importância desse campo de estudo na literatura acadêmica, o que se reflete em um aumento de 99 \% no número de publicações anuais entre 1989 e 2018. Dado o rigor da metodologia e do foco global do campo de estudo, os resultados do documento facilitam a identificação efetiva dos rumos da pesquisa sobre a implementação do IFRS nas organizações.

PALAVRAS-CHAVE implementação, análise bibliométrica, análise de citações, análise de redes. 


\section{Introducción}

Desde la adopción de manera obligatoria de las Normas Internacionales de Información Financiera -NIIF-, emitidas en 2009 por la InternationalAccounting Standards Board-IASB-, se ha creado un interés por las investigaciones alrededor de la contabilidad. El aumento en las publicaciones científicas sobre las NIIF (que en su inicio se denominaban Normas Internacionales de Contabilidad -NIC-) ha permitido conocer los procesos de implementación a nivel mundial, asociando beneficios $y$ dificultades en las organizaciones.

Por ello Mattessich (2012) menciona que el abordaje de estudios pertinentes alrededor de la contabilidad a nivel mundial permite conocer las dimensiones que ha tenido la historia contable, dejando de ser vista como una práctica netamente teórica para convertirse en un verdadero sistema de información útil para el desarrollo de servicios con objetivos prácticos. Así que la aplicación de las NIIF ha cambiado la visión desde acciones netamente teóricas a una herramienta que brinda información a la gerencia para la toma de decisiones.

Distintos estudios relacionan el crecimiento económico de las organizaciones con los manejos de los sistemas contables. Al entender que la gestión contable es considerada como un sistema de información que mide, procesa y comunica información financiera mediante el registro y almacenamiento de datos que son procesados y analizados para brindar información a los tomadores de decisiones. Estos usuarios toman acciones que pueden afectar de manera positiva o negativa la actividad organizacional a través de aspectos tales como calidad de la información, reportes financieros y mejoras en los procesos operativos (Belvert, Powers and Crosson, 2009).

Por su parte Fuentes, García y Toscano (2018) describen que los estudios sobre las NIIF y las NIC a nivel mundial en las organizaciones se convierten en un insumo esencial para revelar los enfoques gerenciales y financieros asociados a las prácticas contables como herramienta administrativa que aporta a la gerencia información fundamental para la toma de decisiones, destacando: (i) los motivadores sociales de la implementación de las NIIF; (ii) los efectos de las NIIF en la gestión financiera y comercial; (iii) las estrategias organizacionales de crecimiento económico y (iv) los factores asociados a calidad de la información (Rudzani and Manda, 2016).

En este contexto, y debido al crecimiento de la producción científica relacionada con la gestión contable (Merigó and Yang, 2017), las síntesis de múltiples investigaciones resultan significativamente útiles como herramientas eficaces para acceder a los recientes desarrollos de la investigación en este campo de estudio (Toscano-Hernández, Álvarez-González y SanzoPérez, 2018). Este es el caso de los estudios bibliométricos que como metodología de investigación del campo de los metaestudios reflexivos de la literatura facilitan obtener una visión general de la estructura y las tendencias de los campos de estudio (Pipere, Veisson and Salite, 2015).

De hecho, en los últimos años, se han realizado numerosos estudios bibliométricos en diversas perspectivas y contextos de la contabilidad. No obstante, ninguno de ellos enfocado en las tendencias de las NIIF y las NIC a nivel mundial. Concretamente entre los estudios bibliométricos en el campo de estudio de la contabilidad se identifican los relacionados con la gestión contable sostenible (Zyznarska-Dworczak, 2018), la investigación contable (Merigó and Yang, 2017), así como la contabilidad social y ambiental (Fusco and Ricci, 2018; Schaltegger, Gibassier and Zvesdov, 2013; Toscano et al., 2018; Yamaguchi, Silva dos Santos and Watanabe, 2015).

En consecuencia, considerando el creciente interés académico y profesional sobre la contabilidad desde diferentes perspectivas, se hacen necesarias las síntesis de múltiples investigaciones originales como instrumentos útiles para acceder a los avances recientes en este campo de estudio.

A partir de lo descrito, este articulo presenta los resultados de un estudio bibliométrico sobre las investigaciones científicas relacionadas con la implementación de las NIIF y las NIC. La metodología se sustenta en publicaciones de revistas científicas indexadas en la base de datos Scopus y analizadas bajo el software VOSviewer que facilita la visualización de mapas 
de red basados en datos bibliográficos (PerianesRodriguez, Waltman and van Eck, 2016), lo que resulta en un aporte significativo y novedoso en este campo de estudio. De esta manera el propósito de este documento es contribuir a la comprensión de la implementación contable mediante un acercamiento a la visión mundial de adopción en las organizaciones a través de los autores con mayores publicaciones y a la vez la evolución de los documentos en revistas de alto impacto.

El objetivo general es el de identificar las principales características bibliográficas de la producción científica mundial relacionada con la implementación de las NIIF y las NIC en las organizaciones a partir de un estudio bibliométrico. El estudio aborda tres objetivos específicos: (i) analizar la evolución temporal, citaciones, tipos de documentos y revistas de la producción científica mundial relacionadas con la implementación de las NIIF y las NIC; (ii) determinar las redes de colaboración internacional en la investigación científica relacionadas con la implementación de las NIIF y las NIC y (iii) explorar los clústeres temáticos en la investigación científica relacionada con la implementación de las NIIF y las NIC.

\section{Marco referencial}

Los análisis bibliométricos durante los últimos años han sido utilizados como herramienta en los campos académicos e investigativos. La importancia de las publicaciones científicas a nivel mundial ha llevado a generar indicadores de medición de autores, revistas, citaciones, países con mayores publicaciones, instituciones con mayores aportes, todos ellos como fuente de análisis de calidad y repercusión en la comunidad científica (Nightingale and Marshall, 2013). La principal ventaja que ofrece el análisis bibliométrico es la de suministrar un cuadro general de un tema de investigación. Esto se convierte en un valor útil para identificar autores, temáticas, revistas, clúster y las principales tendencias en un período proyectado por el investigador (Toscano et al., 2018).
Por ello los estudios de bibliometría proporcionan una completa visión del concepto desde una perspectiva general y en algunos casos proporcionan una imagen puntual de un área de investigación, lo que es muy útil para identificar a los actores más influyentes (Bar-Ilan, 2008). De ahí que los análisis en este campo son constituidos por una aplicación de técnicas y métodos que analizan grandes volúmenes de información para sintetizar resultados que dan un gran aporte a la comunidad científica en los procesos de desarrollo científico y tecnológico (Santos, 2012).

De lo anterior, se resalta que los análisis bibliométricos son herramientas esenciales para el desarrollo de procesos investigativos en las comunidades académicas y para generar una perspectiva de las tendencias en las variables de estudio previo a la inmersión en el desarrollo de un determinado problema; de igual manera ofrece al investigador una visión amplia del comportamiento del objeto de estudio a nivel mundial, detallando indicadores que favorecen el desarrollo conceptual y práctico. A la vez es preciso describir que este tipo de estudio no solo puede ser útil en la comunidad académica, también existe interés de los sectores empresariales en conocer indicadores relacionados con ventas, proveedores, tipos de cliente, tendencias de mercado, preferencias de los clientes entre otros. Así pues, estos indicadores revelan insumos que permiten crear estrategias empresariales en la generación de valor en las organizaciones.

En cuanto a los antecedentes se han identificado algunos documentos relacionados con los análisis documentales y bibliométricos que proporcionan valor a esta investigación, a saber: (i) revisiones documentales con enfoque teórico en el campo de la contabilidad que se centran en conceptos, evolución, uso de la información contable, postura de autores, crecimiento de publicaciones e impacto en la gestión contable. $\mathrm{Su}$ característica principal es la revisión doctrinal y el enfoque axiológico e histórico de la contabilidad; aquí destacan autores tales como Dyckman y Zeff (2006) y Unegbu (2014); (ii) estudios con enfoques bibliométricos en el campo de las publicaciones científicas en contabilidad. Estos se caracterizan por ser estudios cuantitativos cuyo objetivo es examinar los principales resultados de producción científica en el campo contable a través del análisis de bases de datos de alto impacto 
como Web of Science, Scopus y Google Scholar donde destacan indicadores de crecimiento, mayor número de publicaciones, autores con mayor número de publicaciones y citaciones, enfoque de temáticas de estudio e instituciones con mayor productividad científica; (iii) estudios bibliométricos con utilización de herramientas y software especializados en el campo contable para analizar indicadores como, por ejemplo, evolución de publicaciones por período, distribuciones porcentuales de las publicaciones, número de citaciones porcentuales por autores y revista, redes de autores más relevantes de acuerdo a sus citaciones, redes de organizaciones más relevantes, redes de palabras clave y clúster temáticos; (iv) estudios bibliométricos en el campo práctico de la contabilidad, estos se encuentran relacionados con investigaciones que hacen uso del análisis bibliométrico como apoyo conceptual y teórico para entender la problemática desde una visión más holística. Su objetivo principal es el de conocer las perspectivas globales de estudio para darle función en el contexto propio; aquí destacan autores tales como Hulle, Kaspar y Moller (2011), Schaltegger et al. (2013), Özmen (2009) y Yamaguchi, Silva dos Santos y Watanabe (2015).

\section{Metodología}

Este trabajo realiza una descripción de las principales características bibliográficas de la producción científica mundial relacionada con la implementación de las NIIF y las NIC en las organizaciones a partir de un estudio bibliométrico. De hecho, a pesar de que estos análisis son sumamente desafiantes en áreas altamente interdisciplinarias y emergentes (Buter and van Raan, 2013), los estudios bibliométricos evidencian una metodología bien establecida de investigación y bastante frecuente que analiza datos bibliográficos (Pipere et al., 2015). En consecuencia, estos estudios son adecuados para investigar y proporcionar valiosas evaluaciones de las principales tendencias y obtener así una visión general de la estructura intelectual de los campos de estudio (Pipere et al., 2015).

Ahora bien, este estudio bibliométrico se desarrolló siguiendo el procedimiento planteado a través de los principios de la revisión sistemática de literatura - RSL- como un método riguroso y explícito de revisión bibliográfica (Tranfield, Denyer and Smart, 2003). Estos principios de la RSL son el resultado de un consenso razonable que ha surgido en cuanto a las características metodológicas deseables para reportar los resultados de trabajos de revisión de literatura basados en pasos replicables y transparentes (Moher et al., 2009; Tranfield et al., 2003), los cuales establecen unos criterios que pueden ser aplicados por cualquier investigador al mismo resultado. En concreto, la RSL se puede desarrollar a partir de una lista de etapas: (i) etapa de planeación de la revisión que incluye la identificación de la revisión y la preparación de una propuesta de revisión; (ii) etapa de ejecución de la revisión que incluye la identificación de la investigación, selección de estudios, extracción de datos y síntesis de datos; (iii) etapa de informe de la revisión que incluye presentación de resultados y recomendaciones (Tranfield et al., 2003).

Este procedimiento se desarrolló bajo los principios de la RSL, así como la propuesta metodológica de Moher et al. (2009) y el trabajo de Toscano-Hernández, Álvarez-González y SanzoPérez (2018). De esta forma, a continuación, se describe el procedimiento utilizado para desarrollar este estudio bibliométrico que considera tres etapas principales: planeación; ejecución y resultados.

\section{Planeación}

Inicialmente, en la primera etapa, se definen los objetivos del estudio bibliométrico, se identifican las fuentes de información y se precisan los criterios para la identificación de la investigación científica.

\section{Fuente de información}

De acuerdo con los objetivos de este trabajo, se identifica como fuente de búsqueda de información a la base de datos Scopus de Elsevier. Se utiliza como fuente de información 
a Scopus dado que es considerada la base con mayor cobertura a nivel mundial, con estándares de indexación más exigentes y una poderosa capacidad de análisis de citaciones (Bartels, 2013; Falagas et al., 2008). Esto garantiza una mayor cobertura en el estudio bibliométrico y minimiza el sesgo que origina la búsqueda en bases de datos específicas.

\section{Estrategia de búsqueda}

Al considerar los objetivos del presente trabajo, se consideran los documentos de la producción científica mundial relacionados con la implementación de las NIIF y las NIC publicados en fuentes indexadas en Scopus. De igual manera se opta por limitar la búsqueda de producción científica publicada a partir de 1989, primera publicación en el campo, restringiéndola a los campos temáticos de: negocios; economía; finanzas; contabilidad y ciencias sociales.

En este estudio bibliométrico, en coherencia con lo expuesto en el párrafo anterior, se valoraron los términos de búsqueda: "implementation", "IFRS" (NIIF, en inglés) y "IAS" (NIC, en inglés), junto con sus equivalentes. Para fines de búsqueda se dispuso la verificación de la presencia de dichos términos en títulos, resúmenes y palabras clave de las publicaciones. En consecuencia, se realizó la búsqueda de las publicaciones con la siguiente expresión: ((TITLE-ABS-KEY ("implementation" OR "adoption" OR "introduction") AND TITLEABS-KEY ("IFRS" OR "International Financial Reporting Standard" OR "International Accounting Standards" OR "IAS")) AND ((LIMITTO (SUBJAREA, "BUSI") OR LIMIT-TO (SUBJAREA, "ECON") OR LIMIT-TO (SUBJAREA, "SOCI")).

\section{Ejecución}

Seguidamente, en la segunda etapa, se desarrollan los pasos de identificación de la producción científica mundial relacionada con la implementación de las NIIF y las NIC, así como la selección de las publicaciones de mayor relevancia.

\section{Identificación de la producción científica}

En junio de 2018 se ejecuta la estrategia de búsqueda descrita con anterioridad. En efecto, como resultado de la aplicación de la estrategia de búsqueda se logró la identificación de un total de 1224 referencias de publicaciones científicas relacionadas con la implementación de las NIIF y las NIC entre 1989 y junio de 2018.

\section{Selección de las publicaciones de mayor relevancia}

Paralelamente, entre las 1224 referencias de publicaciones científicas relacionadas con la implementación de las NIIF y las NIC identificadas, se propone la selección de las publicaciones científicas de mayor relevancia en la producción científica mundial con el fin de facilitar el análisis de sus datos bibliográficos. De esta manera se seleccionaron las publicaciones indexadas en Scopus que cumplieran los siguientes criterios: (i) artículos científicos; (ii) publicados en revistas científicas; (iii) escritos originalmente en inglés y (iv) publicados entre 1989 y junio de 2018. En consecuencia, se seleccionó un total de 994 publicaciones de mayor relevancia en la producción científica mundial relacionada con la implementación de las NIIF y las NIC.

Se debe señalar que se limitó el estudio a los documentos escritos originalmente en inglés para garantizar un alcance global. Esto se explica por el rol primordial que desempeña este idioma en la investigación científica mundial (Schaltegger et al., 2013) y a que los países de habla inglesa han evidenciado resultados fuertes en la investigación contable (Merigó and Yang, 2017). Así las cosas, junto al francés y al alemán, el inglés es considerado uno de los tres idiomas principales por su cobertura de la población de la Unión Europea y de los países de la OCDE (Schaltegger et al., 2013); asimismo, de las 100 instituciones de investigación con mayor influencia a nivel mundial 
casi todas son de habla inglesa (Merigó and Yang, 2017). De hecho, en la investigación contable "los países que no hablan inglés [...] han mostrado resultados muy débiles, pero quizás hayan publicado excelentes resultados de investigación en sus propios idiomas" (Merigó and Yang, 2017, p. 97).

\section{Reporte}

Por último, en la tercera etapa, se desarrolló el reporte de los resultados del estudio bibliométrico de la producción científica identificada por medio de lo descrito en los dos subapartados anteriores. El reporte de los resultados obtenidos, a partir de la información bibliográfica extraída de las 1224 publicaciones científicas relacionadas con la implementación de las NIIF y las NIC entre 1989 y junio de 2018, se presenta en cuatro partes descritas a continuación.

En la primera parte se presenta un análisis descriptivo de la producción científica mundial relacionada a través de una caracterización cuantitativa de las 1224 publicaciones científicas identificadas donde se considera el año de publicación, el tipo de documentos y el número de citaciones registradas hasta junio de 2018. En la segunda parte se muestran las principales características de las 994 publicaciones de mayor relevancia en la producción científica mundial relacionada por medio de una descripción de la evolución temporal. En la tercera parte se describen las principales redes de colaboración de las 994 publicaciones de mayor relevancia en la producción científica mundial relacionada a partir de un análisis de coautoría que incluye autores, organizaciones y países. En la tercera parte se exponen los principales clústeres temáticos de las 994 publicaciones de mayor relevancia en la producción científica mundial relacionada a partir de un análisis de coocurrencia de sus palabras clave.

Se debe señalar que en los análisis de coautoría y coocurrencia se utilizan herramientas bibliométricas a partir de la construcción de mapas de red basados en datos bibliográficos de publicaciones, los cuales son conocidos como mapas bibliométricos. Estos mapas proporcionan una representación gráfica de las redes de relación entre los términos más comunes en la descripción de los registros bibliográficos de las publicaciones (Perianes-Rodriguez et al., 2016), por lo que son especialmente útiles en la identificación de grupos de elementos relacionados entre publicaciones científicas (Perianes-Rodriguez et al., 2016; van Eck and Waltman, 2010). Dada la funcionalidad del software VOSviewer para la visualización de mapas bibliométricos de una manera fácil de interpretar (Perianes-Rodriguez et al., 2016), estos se construyen y analizan a través de dicho software.

\section{Resultados}

\section{Análisis descriptivo de la producción científica}

En la literatura científica a nivel mundial, a partir de lo descrito en el apartado metodológico, se logró la identificación de un total de 1224 publicaciones científicas relacionadas con la implementación de las NIIF y las NIC entre 1989 y junio de 2018. Se debe señalar que los resultados de las publicaciones de 2018, al ser solo de los primeros seis meses, no son adecuados para compararlos con los de los demás años.

En la figura 1 se evidencia un incremento significativo registrado en el número de publicaciones anuales en el período de observación al pasar de 1 a 140 documentos entre 1989 y 2017 , mientras que se registraron 73 publicaciones tan solo en la primera mitad de 2018. De hecho, entre 1989 y 2017 se registra un promedio anual de 48 publicaciones y una variación porcentual anual en el número de publicaciones del $43 \%$. Sin embargo entre 1989 y 1999 solo se registró un total de 7 publicaciones, lo que significa menos de 1 publicación anual en promedio. Entretanto entre 2000 y 2004 se registran menos de 10 publicaciones anuales, a excepción de 2002 cuando se registraron 11 publicaciones relacionadas. Posteriormente a partir de 2005 se presenta un crecimiento significativo sostenido en el número de publicaciones anuales, a excepción de 2009 y 2014 , siendo 2016 el año de mayor crecimiento en lo que se refiere a producción científica. 


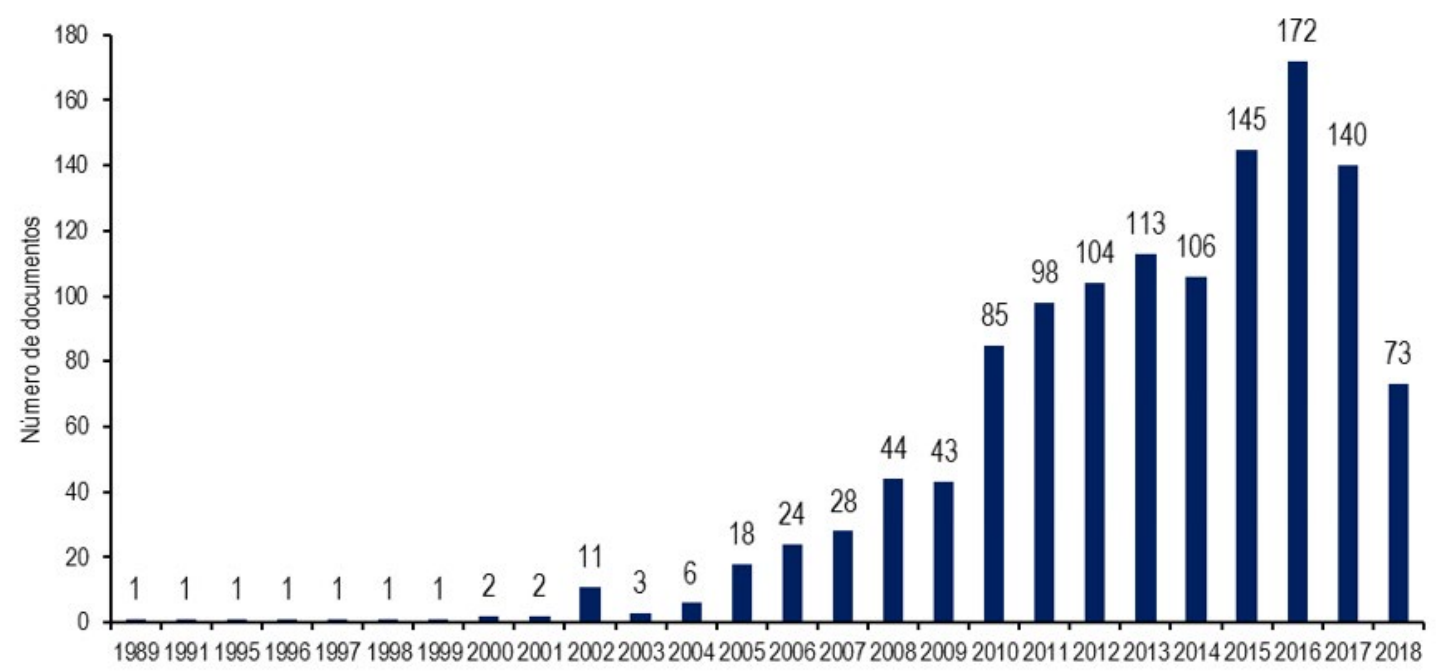

Figura 1. Número de documentos de la producción científica relacionada con la implementación de las NIIF y las NIC entre 1989 y 2018. Fuente: elaboración propia por parte de los autores.

Entre las 1224 publicaciones científicas relacionadas se incluyen documentos de conferencias, libros, capítulos de libro, revisiones bibliográficas, material editorial, cartas a editores y artículos publicados en revistas científicas (tabla 1).

Al analizar la distribución porcentual por tipo de documento identificado se destaca como los artículos científicos representan el mayor porcentaje, pues registran el 84,2 \% de las publicaciones, seguidos por revisiones bibliográficas y capítulos de libro con un 4,2 \% y un 3,7 \% respectivamente. Con relación al número de citaciones registradas hasta junio de 2018 por las 1224 publicaciones científicas relacionadas se observa como los artículos científicos registraron el mayor porcentaje al concentrar el 86,4 \% del total de citas, mientras las revisiones bibliográficas acumularon el $8 \%$ de las mismas.

Tabla 1. Distribución porcentual de la producción científica relacionada con la implementación de las NIIF y las NIC entre 1989 y 2018

\begin{tabular}{|c|c|c|c|c|}
\hline \multirow[t]{2}{*}{ Tipos de documentos } & \multicolumn{2}{|c|}{ Documentos } & \multicolumn{2}{|c|}{ Citaciones } \\
\hline & Número & $\%$ & Número & $\%$ \\
\hline Artículos científicos & 1031 & $84,2 \%$ & 11855 & $86,4 \%$ \\
\hline Artículos en prensa & 25 & $2,0 \%$ & & \\
\hline Libro & 11 & $0,9 \%$ & 42 & $0,3 \%$ \\
\hline Capítulo de libro & 45 & $3,7 \%$ & 68 & $0,5 \%$ \\
\hline Documento de conferencia & 36 & $2,9 \%$ & 551 & $4,0 \%$ \\
\hline Revisiones de conferencias & 2 & $0,2 \%$ & & \\
\hline Editorial & 4 & $0,3 \%$ & 1 & $0,0 \%$ \\
\hline Errata & 1 & $0,1 \%$ & & \\
\hline Carta & 1 & $0,1 \%$ & & \\
\hline Notas & 16 & $1,3 \%$ & 67 & $0,5 \%$ \\
\hline Revisiones & 52 & $4,2 \%$ & 1131 & $8,2 \%$ \\
\hline Total general & 1224 & $100 \%$ & 13715 & $100 \%$ \\
\hline
\end{tabular}

Fuente: elaboración propia por parte de los autores. 


\section{Principales características de las publicaciones de mayor relevancia}

Con base en las 994 publicaciones de mayor relevancia en la producción científica mundial relacionada, seleccionadas de acuerdo a lo explicado con anterioridad, se presenta una descripción de la evolución temporal y de las revistas científicas de estas publicaciones.

Enlafigura 2seexpone la evolución cuantitativa de las publicaciones de mayor relevancia, lo que pone de manifiesto el aumento en el número de artículos científicos sobre la implementación de las NIIF y las NIC en las organizaciones. En el análisis temporal de los artículos científicos, y teniendo en cuenta que en 2018 tan solo se tiene información hasta el mes de junio, se observa un promedio de 41 publicaciones por año y un $48 \%$ de variación porcentual promedio en el número anual de publicaciones relevantes entre $1989 \mathrm{y}$ 2017.
Los resultados de este estudio bibliométrico evidencian como las publicaciones de mayor relevancia en la producción científica relacionada fueron publicadas en un total de 235 revistas científicas (tabla 2). Entre estas 235 revistas donde se ubicaron las publicaciones de mayor relevancia, las 10 revistas con el mayor número de publicaciones concentran un 28,9\%; mientras el $71,1 \%$ restante de las publicaciones de mayor relevancia se distribuye entre las otras 225 revistas. Aunque al comparar la concentración de publicaciones de mayor relevancia por período de tiempo se evidencia que las 225 revistas con el menor número de publicaciones relevantes agrupan solo el 57 y el $64 \%$ de las publicaciones en los períodos de 1989-2000 y 2001-2018 respectivamente. Vale la pena señalar como la revista con el mayor número de publicaciones, Australian Accounting Review, tan solo registra su primera publicación relacionada a partir de 2006.

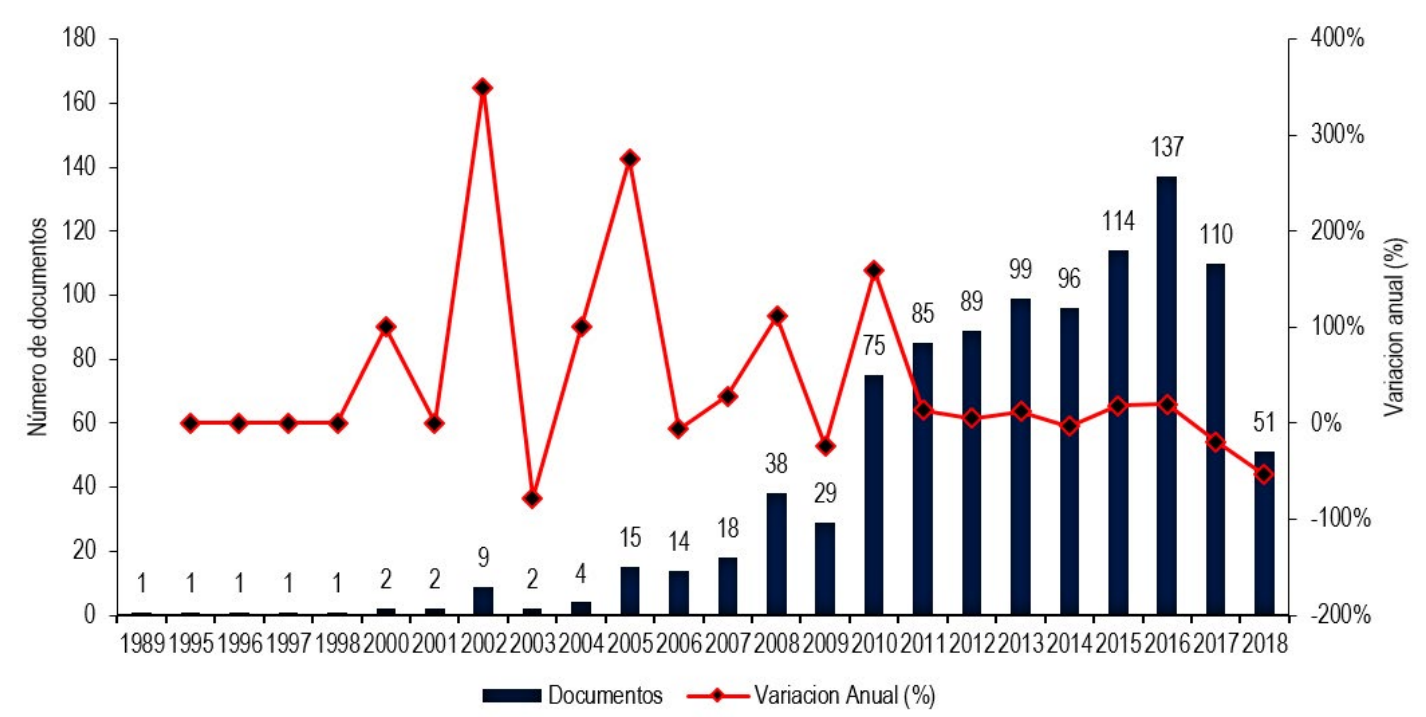

Figura 2. Evolución temporal de la producción científica relacionada con la implementación de las NIIF y las NIC entre 1989 y 2018. Fuente: elaboración propia por parte de los autores. 
Tabla 2. Evolución temporal de la producción científica relacionada con la implementación de las NIIF y las NIC entre 1989 y 2018

\begin{tabular}{|c|c|c|c|c|c|}
\hline \multirow[t]{2}{*}{ Nombre de las revistas } & \multirow[t]{2}{*}{$1989-2000$} & \multirow[t]{2}{*}{ 2001-2010 } & \multirow[t]{2}{*}{ 2011-2018 } & \multicolumn{2}{|c|}{ Total } \\
\hline & & & & Documento & $\%$ \\
\hline Australian Accounting Review & 0 & 14 & 25 & 39 & $3,9 \%$ \\
\hline The International Journal of Accounting & 2 & 9 & 26 & 37 & $3,7 \%$ \\
\hline Accounting in Europe & 0 & 5 & 31 & 36 & $3,6 \%$ \\
\hline Advances in Accounting & 0 & 12 & 20 & 32 & $3,2 \%$ \\
\hline $\begin{array}{l}\text { Journal of International Accounting, } \\
\text { Auditing and Taxation }\end{array}$ & 0 & 10 & 19 & 29 & $2,9 \%$ \\
\hline European Accounting Review & 1 & 14 & 11 & 26 & $2,6 \%$ \\
\hline $\begin{array}{l}\text { Journal of International Accounting } \\
\text { Research }\end{array}$ & 0 & 2 & 22 & 24 & $2,4 \%$ \\
\hline Journal of Applied Business Research & 0 & 2 & 20 & 22 & $2,2 \%$ \\
\hline Accounting and Finances & 0 & 5 & 16 & 21 & $2,1 \%$ \\
\hline $\begin{array}{l}\text { Academy of Accounting and Financial } \\
\text { Studies Journal }\end{array}$ & 0 & 1 & 20 & 21 & $2,1 \%$ \\
\hline Otras 225 & 4 & 132 & 571 & 707 & $71,1 \%$ \\
\hline Total general & 7 & 206 & 781 & 994 & $100 \%$ \\
\hline
\end{tabular}

Fuente: elaboración propia por parte de los autores.

En el análisis de coautoría de las 994 publicaciones de mayor relevancia identificadas se evidencia la existencia de un total de 1753 autores, asociados a 1666 organizaciones (instituciones, universidades, centros de investigación entre otros) de 98 países. Por un lado, a pesar de que fueron identificados un total de 1752 autores con al menos una publicación, los 118 autores con tres publicaciones y una citación fueron agrupados en 53 clúster que en su mayoría reúnen a los coautores de una publicación relevante (figura 3). De hecho, 23 de los 53 clúster están conformados por un solo autor. La mayoría de estos clúster no se encuentran relacionados entre sí, a excepción de los tres clúster que agrupan a los siguientes autores: Brown, P.; Tarca, A.; Haller, A.; Morris, R.D.; Hellman, N.; Gray, S.J.; Lin, Z.; Street, D.L.; Cereola, S.J. y Nichols, N.B. 


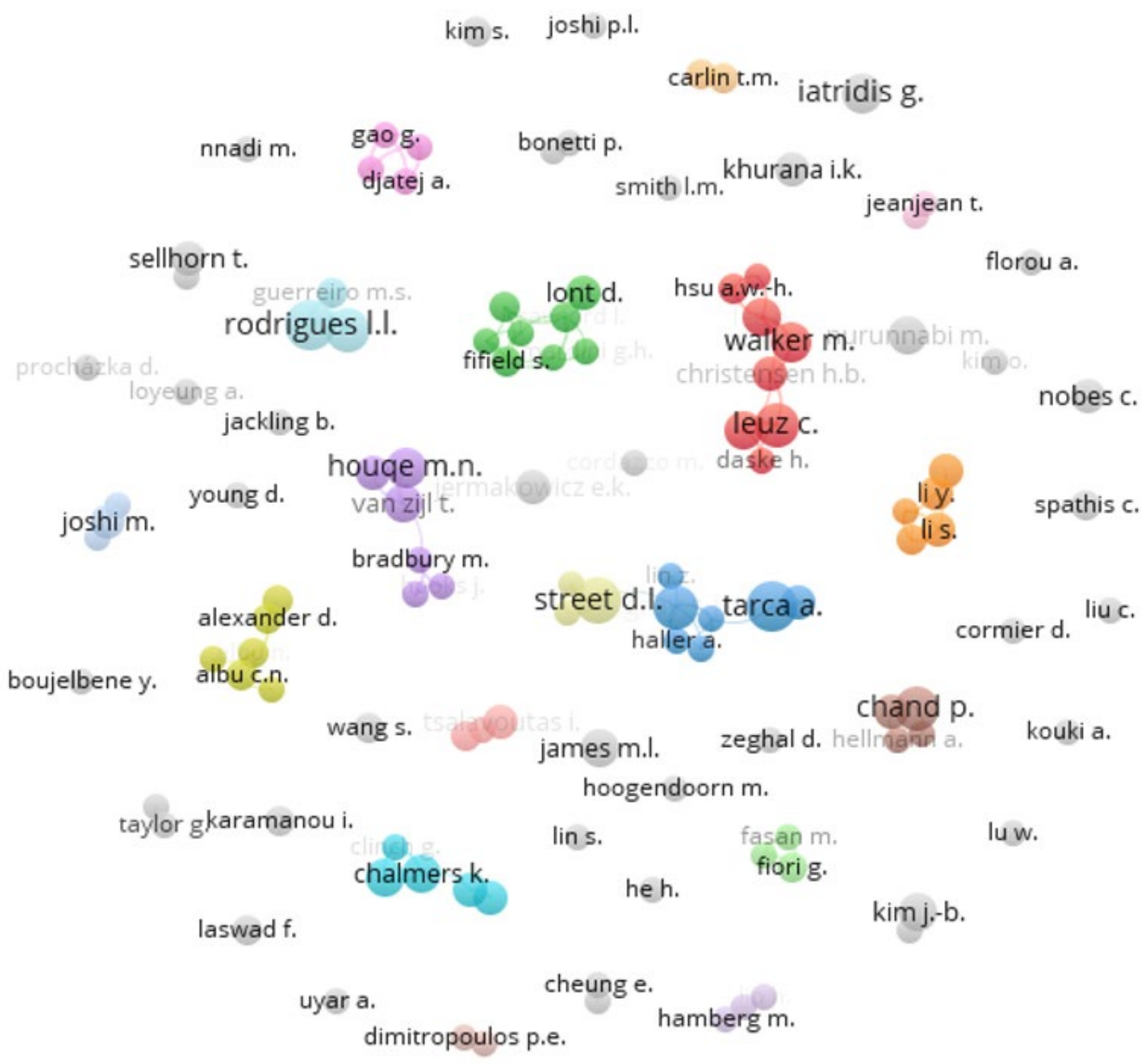

Figura 3. Red de autores de la producción científica relacionada con la implementación de las NIIF y las NIC entre 1989 y 2018. Fuente: elaboración propia por parte de los autores.

De igual manera se identifican cinco clústeres con una coocurrencia superior al resto, que agrupan al menos a cinco autores, conformados por: (i) Chan, A.L., Christensen, H.B., Daske, H., Hail, L., Hsu, A.W., Lee, E., Leuz, C. y Walker, M.; (ii) Crawford, I., Dunne, T., Fifield, S., Hellliar, C., Lont, D., Mardini, G.H. y Power, D.; (iii) Brown, P., Gray, S.J., Haller, A., Hellman, N., Lin, Z., Morris, R.D. y Tarca, A.; (iv) Albu, N., Alexander, D., Alon, A., Hodgson A. y Strouhal, J.; (v) Bradbury, M., Hooks, J., Houqe, M.N., Monem, R.M., Stent, W. y van Zijl, T.

Por otro lado se puede observar la red de 100 organizaciones con al menos dos documentos y una citación, agrupadas en 65 clúster (figura 4). Se debe mencionar que 42 de los 65 clúster de organizaciones están conformados por una sola organización y solo 7 organizaciones están relacionadas entre sí, a partir de coautorías de artículos relevantes. En efecto, solo seis organizacionesregistrancincoomáspublicaciones: Cal State University (Estados Unidos); Macquarie University (Australia); School of Accounting of the RMIT University (Australia); University of New South Wales (Australia); University of Southern California (Estados Unidos) y The University of Western Australia (Australia). Entretanto solo 16 organizaciones registran un mínimo de 100 citaciones, de ellas solo cinco registran al menos 200 citaciones: Santa Clara University (Estados Unidos); University of Southern California (Estados Unidos); The Chinese University of Hong Kong (Hong Kong); University of Miami (Estados Unidos) y University of Pennsylvania (Estados Unidos). Adicionalmente, a pesar de que se identifican un total de 82 países con artículos relevantes, tan solo 58 países registran un mínimo de dos publicaciones y una citación (figura 5). 
kazan federal university, inst

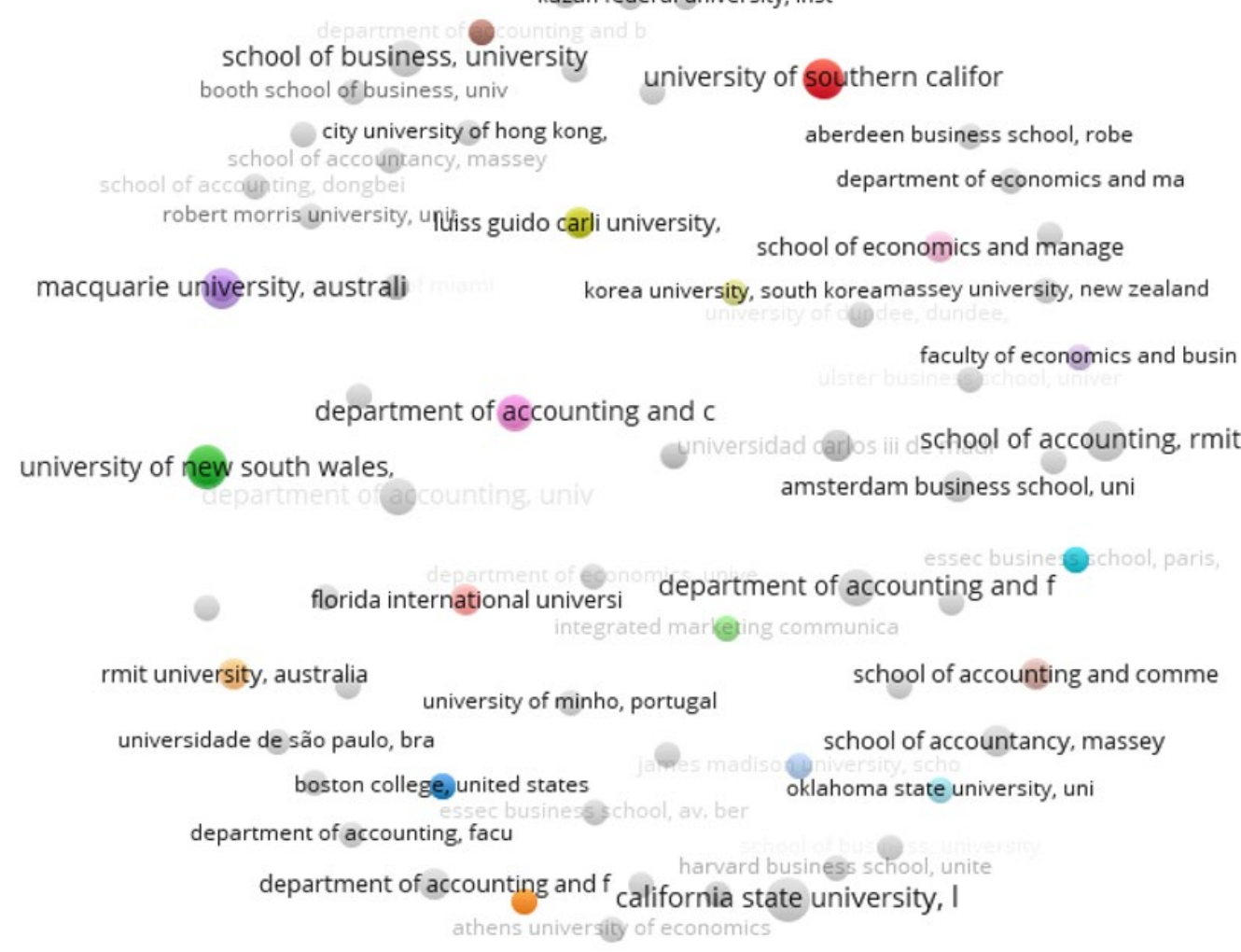

Figura 4. Red de organizaciones con autores de la producción científica relacionada con la implementación de las NIIF y las NIC entre 1989 y 2018. Fuente: elaboración propia por parte de los autores.

Asimismo, se resaltan los 10 países involucrados en el mayor número de publicaciones: Estados Unidos (221 documentos); Australia (144 documentos); Reino Unido (115 documentos); Italia (47 documentos); Canadá (46 documentos); Grecia (45 documentos); Nueva Zelanda (44 documentos); España (42 documentos); Alemania (39 documentos) y Corea del Sur (35 documentos). Se debe señalar que en la figura 5 se aprecia la significativa relación entre los 58 países con al menos dos publicaciones y una citación, agrupados en 13 clúster de países con estrecha relación, a excepción de cinco países que no están relacionados entre sí por publicaciones conjuntas con otros países (Chile, Eslovaquia, Ucrania, Dinamarca y Croacia). 


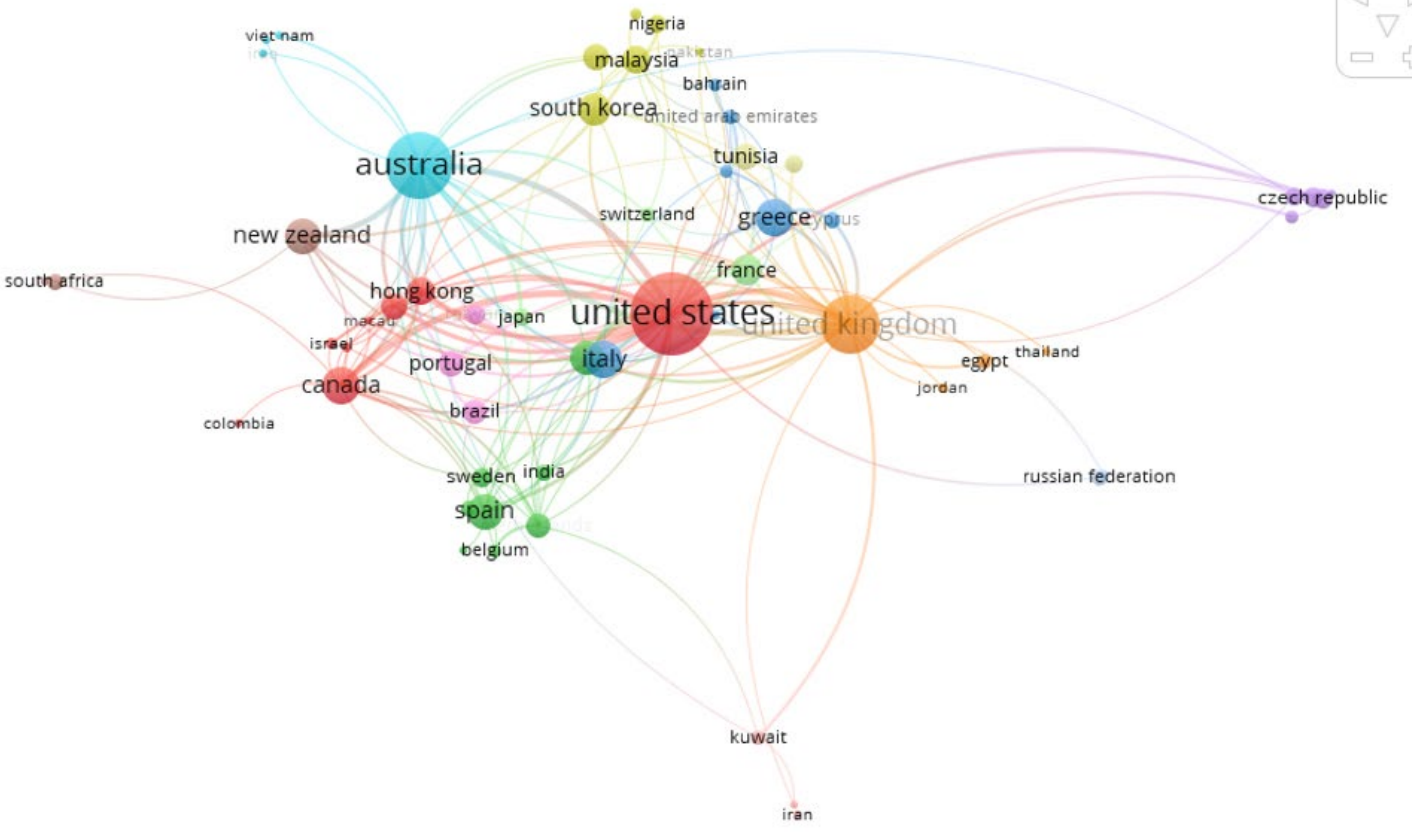

Figura 5. Red de palabras clave de la producción científica relacionada con la implementación de las NIIF y las NIC entre 1989 y 2018. Fuente: elaboración propia por parte de los autores.

\section{Clúster temáticos}

El análisis de coocurrencia, a partir de las palabras clave de los artículos de mayor relevancia, permite visualizar la complejidad y variedad de los hallazgos en la investigación científica relacionada. En la figura 6 se muestra la red de 128 palabras clave con mayor ocurrencia, de un total de 2093 palabras clave que caracterizan las publicaciones bajo estudio. Ahora bien, excluyendo las palabras clave incluidas en las expresiones detalladas en la metodología de este trabajo, se destacan las 10 palabras clave con la relación más estrecha y mayor coocurrencia que caracterizan los artículos científicos de mayor relevancia relacionados con la implementación de las NIIF y las NIC en las organizaciones: value relevance; earnings management; corporate governance; earnings quality; disclosure; accounting quality; information asymmetry; convergence; accounting regulation; harmonization-enforcement. 


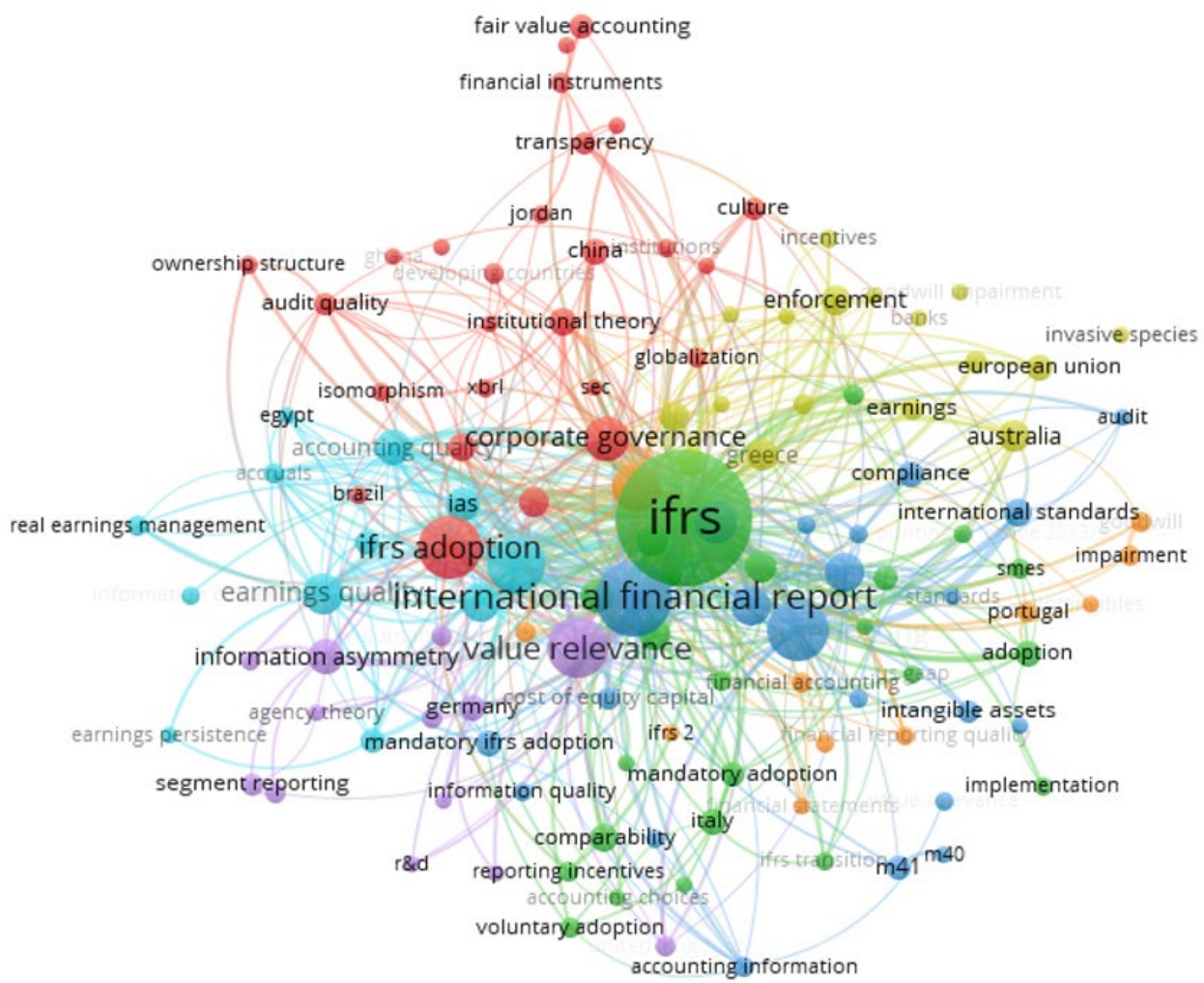

Figura 6. Red de países con autores de la producción científica relacionada con la implementación de las NIIF y las NIC entre 1989 y 2018. Fuente: elaboración propia por parte de los autores.

\section{Discusión y conclusiones}

Los resultados de este estudio bibliométrico evidencian el enorme interés generado en este campo de estudio, lo que se refleja en el significativo crecimiento de la producción científica mundial entre 1989 y 2018. De igual manera debido a la novedosa metodología empleada y al enfoque global del ámbito de estudio, los resultados del presente trabajo se ubican como un importante aporte al estudio de la contabilidad y las finanzas. De hecho, este es el primer estudio bibliométrico que informa sobre las principales redes de colaboración y los clúster temáticos de la producción científica relacionada con la implementación de las NIIF y las NIC en las organizaciones a partir de la producción científica de los últimos 28 años disponible en Scopus.

En concreto, la importancia de este campo de estudio se refleja en la identificación de un total de 1224 publicaciones académicas con 13715 citaciones relacionadas con la implementación de las NIIF y las NIC en las organizaciones entre 1989 y junio de 2018. Se debe mencionar como en este campo de estudio se registra un significativo incremento en el número anual de publicaciones al pasar de 1 a 140 documentos entre 1989 y 2017. No obstante, esto se debe considerar con cautela debido a que entre 1989 y 1999 solo se registra 1 documento anual en este campo de estudio; mientras que entre 2000 y 2004 se registran menos de 10 publicaciones anuales, a excepción de 2002 cuando se registraron 11 publicaciones relacionadas. Tan solo a partir de 2005 se da un crecimiento significativo y sostenido en el número de publicaciones anuales, con excepción de 2009 y 2014, siendo 2016 el de mayor auge en lo que se refiere a la producción científica.

Con todo, el análisis de las redes de colaboración científica y los clústeres temáticos de las publicaciones de mayor relevancia permite visualizar las variedades y las complejidades de la red de colaboración. Por un lado, con relación 
a las redes de colaboración, a partir del análisis de coautoría, se evidencian 994 publicaciones de mayor relevancia identificadas con un total de 1753 autores asociados a 1666 organizaciones de 98 países. Por otro, a través de los clústeres temáticos, a partir del análisis de coocurrencia, en la información bibliográfica de las publicaciones de mayor relevancia, se logro identificar una red de 128 palabras clave con mayor ocurrencia entre un total de 2093 palabras clave que caracterizan a las diversas publicaciones científicas.

Se puede resaltar como Estados Unidos y Australia, junto con sus organizaciones, juegan un papel dominante en la investigación científica relacionada. Entretanto los investigadores que presentan el mayor número de publicaciones $\mathrm{y}$ una mayor cantidad de citaciones como coautores de la producción científica relevante sobre la implementación de las NIIF y las NIC en las organizaciones son: Leuz, C., University of Chicago (Estados Unidos); Hail, L., University of Pennsylvania (Estados Unidos); Li, S., Santa Clara University (Estados Unidos); Tarca, A., The University of Western Australia (Australia); Christensen, H.B., University of Chicago (Estados Unidos); Kim, J.-B., City University of Hong Kong (Hong Kong); Iatridis, G., University of Thessaly (Grecia); Rodrigues, L.L., University of Minho (Portugal); Chalmers, K., Monash University (Australia) y Craig, R., Victoria University (Australia).

En lo referente a las temáticas de investigación preferidas relacionadas con la implementación de las NIIF y las NIC en las organizaciones se puede argumentar que los temas de mayor interés están conectados con los términos que registran una relación más estrecha y una mayor coocurrencia entre las demás palabras clave: value relevance; earnings management; corporate governance; earnings quality $y$ disclosure. De esta manera dichos términos permiten inferir la importancia y potencial futuro de cinco temáticas de estudio o tendencias de investigación relacionadas: (i) el impacto de la adopción de las NIIF y las NIC sobre la valoración razonable de los libros; (ii) los efectos de la adopción de las NIIF y las NIC en la gestión de ganancias; (iii) la relación entre los sistemas de gobierno corporativo y la adopción de las NIIF y las NIC; (iv) los efectos de la adopción de las NIIF y las NIC en la calidad de las ganancias contables y la información de los estados financieros; (v) las consecuencias económicas de la divulgación y la regulación de informes financieros.

Finalmente los resultados de este estudio bibliométrico se deben interpretar con prudencia debido a que están asociados al análisis de una serie de publicaciones identificadas por determinados criterios de selección específicos que, a pesar de esforzarse por ser exhaustivos e incluyentes, en la práctica, no representan totalmente la enorme variedad de contribuciones realizadas a la investigación científica en este campo de estudio. Adicionalmente se utilizó como fuente de búsqueda información de una base de datos que, aunque es reconocida por su alcance mundial y rigor científico, tiene recursos limitados. En consecuencia, para estudios bibliométricos futuros, se sugiere ampliar el alcance de la búsqueda en cuanto al número de bases de datos y tipos de publicaciones.

\section{Referencias}

Bar-Ilan, J. (2008). Informetrics at the beginning of the 21st century-A review. Journal of Informetrics, 2(1), $1-52$.

Bartels, E. (2013). How to perform a systematic search. Best Practice \& Research: Clinical Rheumatology, 27(2), 295-306.

Belvert, N., Powers, M. and Crosson, S. (2009). Principles of accounting. Family Systems Medicine, 2(4), 363-364.

Buter, R.K. and van Raan, A.F.J. (2013). Identification and analysis of the highly cited knowledge base of sustainability science. Sustainability Science, 8(2), 253-267.

Dyckman, T.R. and Zeff, S.A. (2006). Two Decades of the Journal of Accounting Research. Journal of Accounting Research, 22(1), 225-297.

Falagas, M.E. et al. (2008). Comparison of PubMed, Scopus, Web of Science, and Google Scholar: strengths and weaknesses. The FASEB Journal, 22(2), 338-342.

Fuentes, D.D., García, H.A. y Toscano, A.E. (2018). Estado actual de la implementación de las Normas Internacionales de Información Financiera (NIIF) en 
PyMEs de la ciudad de Montería, Colombia. FACES, 24(51), 1-16.

Fusco, F. and Ricci, P. (2018). What is the stock of the situation? A bibliometric analysis on social and environmental accounting research in public sector. International Journal of Public Sector Management, 32(1), 21-41.

Hulle, J., Kaspar, R. and Moller, K. (2011). Multiple Criteria Decision-Making in Management Accounting and Control-State of the Art and Research Perspectives Based on a Bibliometric Study. Journal of Multi-Criteria Decision Analysis, 18(5-6), 253-265.

Mattessich, R. (2012). Academic Research in Accounting: The Last 50 Years. Asia-Pacific Journal of Accounting, 3(1), 3-81.

Merigó, J.M. and Yang, J.-B. (2017). Accounting Research: A Bibliometric Analysis. Australian Accounting Review, 27(1), 71-100.

Moher, D. et al. (2009). Preferred reporting items for systematic reviews and meta-analyses: The PRISMA statement. PLoS Medicine, 6(7), e1000097.

Nightingale, J.M. and Marshall, G. (2013). Reprint of “Citation analysis as a measure of article quality, journal influence and individual researcher performance." Nurse Education in Practice, 13(5), 429-436.

Özmen, Ö. (2009). Business Ethics Research with an Accounting Focus: A Bibliometric Analysis from 1988 to 2007. Journal of Business Ethics, 93(1), 137-160.

Perianes-Rodriguez, A., Waltman, L. and van Eck, N.J. (2016). Constructing bibliometric networks: A comparison between full and fractional counting. Journal of Informetrics, 10(4), 1178-1195.

Pipere, A., Veisson, M. and Salite, I. (2015). Developing Research in Teacher Education for Sustainability: UN DESD via the Journal of Teacher Education for Sustainability. Journal of Teacher Education for Sustainability, 17(2), 5-43.

Rudzani, S. and Manda, D.C. (2016). An assessment of the challenges of adopting and implementing IFRS for SMEs in South Africa. Problems and Perspectives in Management, 14(2-1), 212-221.

Santos, G. (2012). Aproximaciones a un análisis bibliométrico de la Revista Internacional Legis de Contabilidad \& Auditoría 2000-2012. Revista Internacional Legis de Contabilidad \& Auditoria, 50, 183-226.
Schaltegger, S., Gibassier, D. and Zvesdov, D. (2013). Environmental management accounting: a bibliometric literature review. Meditari Accountancy Research, 21(1), 4-31.

Toscano-Hernández, A., Álvarez-González, L.I. y Sanzo-Pérez, M.J. (2018). Tendencias globales de la investigación científica relacionada con la sostenibilidad en la universidad: un análisis bibliométrico 2008-2017. En Álvarez-Arregui, E. et al. (Ed.), Universidad, investigación y conocimiento: comprensión e intervención en una sociedad compleja (pp. 237-244). Oviedo, España: Universidad de Oviedo.

Toscano, A. et al. (2018). Contabilidad ambiental y sostenibilidad en las organizaciones: redes de colaboración y clúster temáticos de la producción científica mundial. En Canabal, J.D. et al., Innovar, estrategia de sostenibilidad para el crecimiento y desarrollo empresarial (pp. 62-78). Montería, Colombia: Universidad del Sinú.

Tranfield, D., Denyer, D. and Smart, P. (2003). Towards a methodology for developing evidence-informed management knowledge by means of systematic review. British Journal of Management, 14(3), 207222.

Unegbu, A.O. (2014). Theories of Accounting: Evolution \& Developments, Income-Determination and Diversities in Use. Research Journal of Finance and Accounting, 5(19), 1-16.

Van Eck, N. and Waltman, L. (2010). Software survey: VOSviewer, a computer program for bibliometric mapping. Scientometrics, 84(2), 523-538.

Yamaguchi, C.K., Silva dos Santos, A.P. and Watanabe, M. (2015). Environmental accounting: A bibliometric study. Espacios, 36(11), 8.

Zyznarska-Dworczak, B. (2018). The Development Perspectives of Sustainable Management Accounting in Central and Eastern European Countries. Sustainability, 10(5), 1-21. 